\title{
Does Water Vapor Prevent Upscaling Sonoluminescence?
}

\author{
Ruediger Toegel, ${ }^{1}$ Bruno Gompf, ${ }^{2}$ Rainer Pecha,${ }^{2}$ and Detlef Lohse ${ }^{1}$ \\ ${ }^{1}$ Department of Applied Physics and J.M. Burgers Centre for Fluid Dynamics, University of Twente, \\ 7500 AE Enschede, The Netherlands \\ ${ }^{2}$ 1. Physikalisches Institut, Universität Stuttgart, Pfaffenwaldring 57, D-70550 Stuttgart, Germany
}

(Received 18 May 2000; revised manuscript received 27 July 2000)

Experimental results for single-bubble sonoluminescence of air bubbles at very low frequency $f=$ $7.1 \mathrm{kHz}$ are presented: In contrast to the predictions of a recent model [S. Hilgenfeldt and D. Lohse, Phys. Rev. Lett. 82, 1036 (1999)], the bubbles are only as bright $\left(10^{4}-10^{5}\right.$ photons per pulse) and the pulses as long $(\approx 150 \mathrm{ps})$ as at $f=20 \mathrm{kHz}$. We can theoretically account for this effect by incorporating water vapor into the model: During the rapid bubble collapse a large amount of water vapor is trapped inside the bubble, resulting in an increased heat capacity and hence lower temperatures, i.e., hindering upscaling. At this low frequency water vapor also dominates the light emission process.

PACS numbers: $78.60 . \mathrm{Mq}$

Upscaling single-bubble sonoluminescence (SBSL) [1-4] is of prime importance both for possible application and for understanding the phenomenon. It has been suggested [5] that lowering the acoustical driving frequency from the standard $f=20-35 \mathrm{kHz}$ to $f \approx 5 \mathrm{kHz}$ should yield a 100 to 1000 times higher gain of light, due to the larger ambient radius $R_{0}$ and the prolonged expansion phase. These numbers are based on the thermal bremsstrahlung model [6-9]; however, water vapor was not taken into consideration. Its relevance for SBSL has been revealed recently [10-13], and its role is of increasing importance at lower frequencies: Water vapor diffuses into the bubble during the expansion and is trapped at the subsequent collapse [13]. The result is an increased heat capacity due to the additional number of particles and correspondingly less heating. As at low driving frequencies the bubble expands to a larger maximal radius and thus has lower internal pressure at maximum, more water vapor can be collected, and its role becomes more pronounced, as already suggested in [14].

In this paper we present experimental results obtained at a frequency of $7.1 \mathrm{kHz}$ which give rise to the idea that the above mechanism sets an upper limit for the maximal temperature achievable in SBSL experiments. We then extend the theoretical model of Refs. $[5,7,14,15]$ by taking the water vapor into consideration. In this way the flux of vapor and its influence on the temperature can be quantitatively described.

Experimental setup and results. - SBSL is achieved in the usual way by trapping an air bubble in a spherical resonator. The volume of this resonator is approximately $6 l$ corresponding to a resonance frequency of $7.1 \mathrm{kHz}$. As predicted in [5] degassing down to about 5\% of the natural air saturation (monitored through the oxygen concentration) is necessary to achieve stable sonoluminescence. The gas concentration is expressed in terms of the relative argon saturation $c_{\mathrm{Ar}} / c_{0, \mathrm{Ar}}$ which is the relevant one for sonoluminescence $[16,17]$ and which originates from the $1 \%$ argon contained in air. The pulse width and the number of photons being emitted were measured by time correlated single photon counting $[18,19]$. Figure 1 displays the result for different gas concentrations and temperatures.

(i) The total number of photons lies around $10^{4}-10^{5}$ per pulse, i.e., roughly the same as at $20 \mathrm{kHz}[18,19]$. (ii) The pulse widths are found to be between $100 \mathrm{ps}$ for the darkest bubbles and $180 \mathrm{ps}$ for the brightest ones. Again, these pulse widths are comparable with pulse widths at $20 \mathrm{kHz}[18,19]$. (iii) Figure 2 displays the autocorrelation of the light flash once measured in the UV regime (300-400 $\mathrm{nm}$ ) and once in the red regime $(590-650 \mathrm{~nm}$ ) for the same bubble (maximum normalized to unity). There is no measurable difference, again as for SBSL bubbles at $20 \mathrm{kHz}[18,19]$.

These results give rise to the idea that the maximum temperature of the bubble, which in the thermal

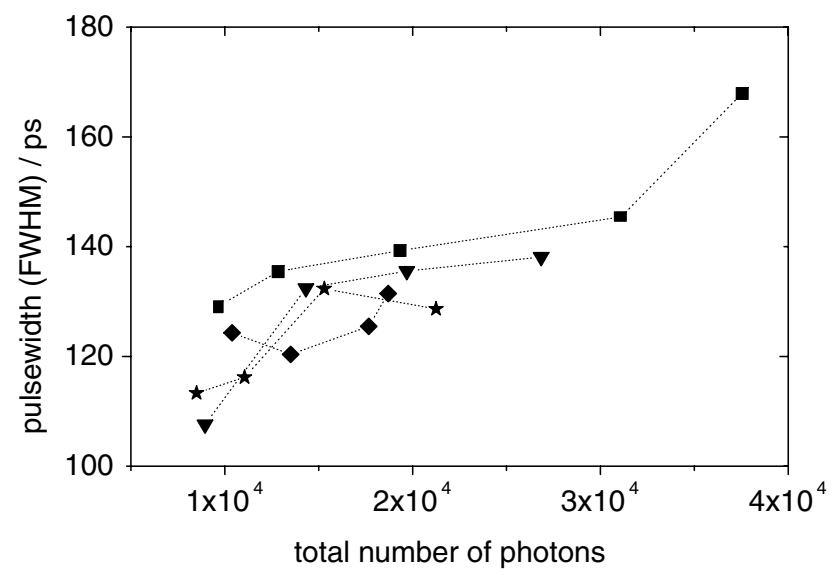

FIG. 1. The pulse width of the light flash for sonoluminescing bubbles at $7.1 \mathrm{kHz}$ as a function of the total number of photons. The relative argon saturation and the liquid temperature are the following: $1.0 \times 10^{-3}$ and $21.9^{\circ} \mathrm{C}$ (squares), $6.3 \times 10^{-4}$ and $19.6^{\circ} \mathrm{C}$ (triangles), $6.2 \times 10^{-4}$ and $22.6^{\circ} \mathrm{C}$ (diamonds), $7.0 \times 10^{-4}$ and $22.8^{\circ} \mathrm{C}$ (stars). Typical pulse widths are around $150 \mathrm{ps}$, typical number of photons around $10^{4}-10^{5}$ per pulse. 
bremsstrahlung model is closely related to the light emission, will also be roughly the same as at $20 \mathrm{kHz}$, i.e., around $15000 \mathrm{~K}$. In contrast, the model of Ref. [5], which neglected water vapor, predicts temperatures beyond $50000 \mathrm{~K}$. We therefore set out to include the effect of water vapor in that model. Clearly, the most precise treatment of sonoluminescing bubbles is through full numerical simulations of the gas dynamical partial differential equations (PDE) as done, e.g., in Refs. $[6,11,13]$, where effects like vapor evaporation and condensation, mass segregation, and even many chemical reactions are considered [20]. The price to pay for these large PDE simulations is that only singular points of the enormous parameter space of SBSL can be calculated. An approximate ordinary differential equation (ODE) approach in the spirit of Refs. $[7,10,14,15]$ as presented here is useful to easily scan the parameter space. We therefore derive an ODE model, which consists of three coupled differential equations, describing the radial motion of the bubble as well as the heat loss and the mass change due to evaporation and condensation of water vapor.

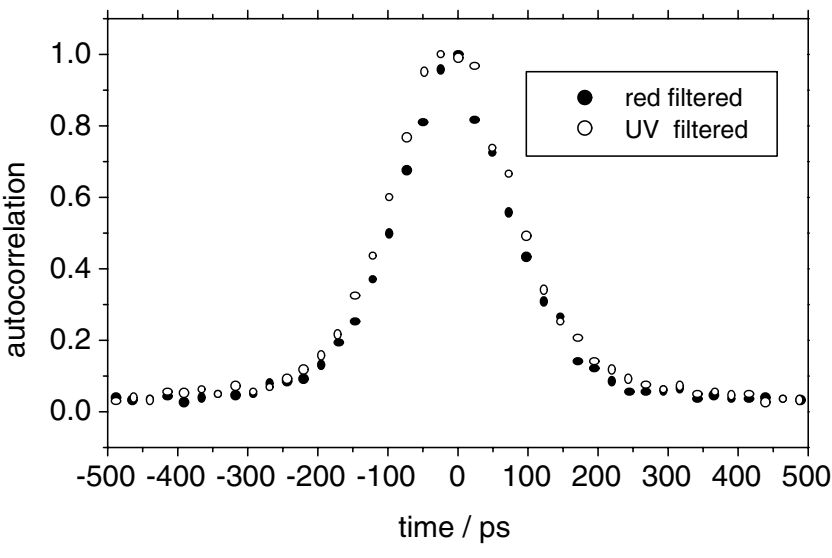

FIG. 2. Direct comparison of the pulse width in the UV $(300-400 \mathrm{~nm})$ and in the red regime $(590-650 \mathrm{~nm})$ of the SL spectrum $\left(c_{\mathrm{Ar}} / c_{0, \mathrm{Ar}}: 5.2 \times 10^{-4}, T_{0}=22^{\circ} \mathrm{C}\right)$. No significant difference in the pulse width is observed.

Bubble dynamics: The bubble motion is described by the Keller-Miksis equation [21,22], which takes sound loss and compressibility effects of the water into account,

$$
\left(1-\frac{\dot{R}}{c_{l}}\right) R \ddot{R}+\frac{3}{2} \dot{R}^{2}\left(1-\frac{\dot{R}}{3 c_{l}}\right)=\left(1+\frac{\dot{R}}{c_{l}}\right) \frac{1}{\rho_{l}}\left(p_{g}-P_{a}-P_{0}\right)+\frac{R}{\rho_{l} c_{l}} \dot{p}_{g}-4 \nu \frac{\dot{R}}{R}-\frac{2 \sigma}{\rho R} .
$$

Here $P_{0}=1 \mathrm{~atm}$ is the ambient pressure and $\rho=$ $1000 \frac{\mathrm{kg}}{\mathrm{m}^{3}}$ the density of water. The viscosity $\nu$, the speed of sound in water $c_{l}$, and the surface tension $\sigma$ are chosen according to the liquid temperature $T_{0}$. For the gas pressure $p_{g}$ and its time derivative a van der Waals type equation of state is used where the total number of particles $N_{\text {tot }}(t)$ is allowed to vary according to condensation and evaporation of water vapor,

$$
p_{g}(t)=\frac{N_{\mathrm{tot}}(t) k T}{\frac{4 \pi}{3}\left[(R(t))^{3}-\left(R_{0}(t) / 8.86\right)^{3}\right]} .
$$

Correspondingly, the equilibrium radius $R_{0}(t)$ under ambient condition is now also time dependent and is another way to express the time dependence of the number of particles $N_{\text {tot }}(t)$ in the bubble. Both are connected by $\frac{4 \pi}{3}\left(P_{0}+\frac{2 \sigma}{R_{0}}\right)\left[\left(R_{0}\right)^{3}-\left(\frac{R_{0}}{8.86}\right)^{3}\right]=N_{\text {tot }} k T_{0}$. Taking a common hard core radius $R_{0} / 8.86$ is justified by the fact that the covolume of argon and water vapor differs by only $5 \%$ [23] and therefore both species can be treated equally.

Mass Diffusion: From Ref. [24] it is known that the surface temperature of the bubble exceeds the water temperature for only a very brief moment during collapse. Therefore, we divide the bubble into two parts, namely, a "cold" boundary layer being in thermal equilibrium with the liquid (this implies condensation to be fast enough to maintain equilibrium) and an eventually hot, homogeneous core. An analysis of the instantaneous diffusive penetration depth $l_{\text {diff }}=\sqrt{R D / \dot{R}}$ shows that two cases occur. During expansion and a major part of the afterbounces the penetration depth exceeds the bubble radius $\left(l_{\text {diff }} \geq R\right)$ implying the total volume to be in equilibrium with the liquid. Dur- ing collapse, however, $l_{\text {diff }}$ becomes as small as $0.01 R$ and the thickness of the boundary layer is negligible as compared to the total bubble volume. Hence in both cases the bubble can be regarded as homogeneous, and we can estimate the rate of particle change to be

$$
\dot{N}_{\mathrm{H}_{2} \mathrm{O}}=\left.4 \pi R^{2} D \partial_{r} n\right|_{r=R} \approx 4 \pi R^{2} D \frac{n_{R}-n}{l_{\mathrm{diff}}} .
$$

$n_{R}=n_{R}\left(T_{0}\right)$ corresponds to the equilibrium density at the wall and $n$ is the actual concentration, $n=N_{\mathrm{H}_{2} \mathrm{O}} / V$.

So far we did not take into account that $l_{\text {diff }}$ cannot exceed a length comparable to the bubble radius $R$, as it would be the case when $\dot{R}$ tends to zero. In this case the convection-diffusion equation for the water vapor inside the bubble becomes a pure diffusion PDE, $\partial_{t}(r n)=$ $D \partial_{r}^{2}(r n)$, with the boundary condition $n(r=R)=n_{R}$. Expressing the solution in terms of a Fourier series, $r(n-$ $\left.n_{R}\right)=\sum_{k=1}^{\infty} a_{k}(t) \sin (k \pi r / R)$, and assuming that the system will be dominated by $k=1$ a characteristic length and hence the desired cutoff is found to be $R / \pi$, thus $l_{\text {diff }}=\min (\sqrt{R D / \dot{R}}, R / \pi)$.

In order to close the equation we have to determine an effective binary diffusion constant $D$ of the mixture. We calculate this quantity - from the kinetic theory of gases [25] — with the properties of the boundary layer, i.e., (i) the water temperature $T_{0}$ and (ii) the number density $n=n_{R}+N_{a r} / V$.

Heat diffusion: Because of $D / \chi \approx 1$ and the complete analogy between thermal and mass diffusion we now apply the same approach as for the water vapor to the temperature field. An analogous equation for the mass balance 
[Eq. (3)] is obtained from the first law of thermodynamics for an open system [26], $d E=h d N_{\mathrm{H}_{2} \mathrm{O}}+d Q-d W$ or

$$
\dot{E}=h \dot{N}_{\mathrm{H}_{2} \mathrm{O}}+\dot{Q}-\dot{W} \text {. }
$$

Here $h=h(p, V, T, N)$ denotes the enthalpy per water molecule, $d Q$ is the heat transferred to the bubble, and $d W$ represents the work done by the bubble.

Internal energy: The internal energy is made up of (i) the translational energy of the argon atoms and (ii) the translational and internal energy of the water molecules,

$$
E=\frac{3}{2} N_{\mathrm{Ar}} k T+\left[\frac{6}{2}+\sum\left(\frac{\theta_{i} / T}{e^{\theta_{i} / T}-1}\right)\right] N_{\mathrm{H}_{2} \mathrm{O}} k T .
$$

$\theta_{i}$ are the characteristic vibrational temperatures [27].

Enthalpy: The water molecules condense at the cold bubble surface. The enthalpy per water molecule is hence given by $h \approx \frac{8}{2} k T_{0}$.

Heat Transfer: Analogously to Eq. (3) the heat loss is estimated to be

$$
\dot{Q}=4 \pi R^{2} \lambda_{\text {mix }} \frac{T_{0}-T}{l_{\text {th }}}, \quad l_{\text {th }}=\min \left(\frac{R}{\pi}, \sqrt{\frac{R \chi}{\dot{R}}}\right) .
$$

In principle, the thermal diffusivity $\chi=\frac{\lambda_{\text {mix }}}{\rho_{\text {mix }} c_{p, \text { mix }}}$ is found from $\rho_{\text {mix }} c_{p, \text { mix }}=\frac{8}{2} n_{R} k+\frac{5}{2} n_{\mathrm{Ar}} k$. Unfortunately, there is no rigorous method to derive an effective thermal conductivity $\lambda_{\text {mix }}$ of a mixture of polyatomic gases. However, a semiempirical expression is given, e.g., in [25]. We strictly follow that approach using for the temperature the liquid temperature $T_{0}$. For further details we refer to [25].

Work: Because of the constant temperature condition at the wall $\left(\lambda_{\mathrm{H}_{2} \mathrm{O}} \gg \lambda_{\text {gas }}\right)$ the latent heat of the water vapor does not contribute to the energy balance and, in particular, the work done by the bubble reduces to the expansion work $\dot{W}=p \dot{V}$.

Now plugging everything together the temperature change of the bubble finally is

$$
\begin{aligned}
\dot{T}= & \frac{\dot{Q}}{C_{v}}-\frac{p \dot{V}}{C_{v}} \\
& +\left[\frac{8}{2} T_{0}-\frac{6}{2} T-T \sum\left(\frac{\theta_{i} / T}{e^{\theta_{i} / T}-1}\right)\right] \frac{\dot{N} k}{C_{v}}, \\
C_{v}= & \frac{3}{2} N_{\mathrm{Ar}} k+\left[\frac{6}{2}+\sum\left(\frac{\left(\theta_{i} / T\right)^{2} e^{\theta_{i} / T}}{\left(e^{\theta_{i} / T}-1\right)^{2}}\right)\right] N_{\mathrm{H}_{2} \mathrm{O}} k .
\end{aligned}
$$

Numerical results. - To verify the model we calculate $R(t), R_{0}(t)$, and $N_{\mathrm{H}_{2} \mathrm{O}}(t)$ for the same parameters $f=$ $26.5 \mathrm{kHz}, R_{0}^{\mathrm{Ar}}=4.5 \mu \mathrm{m}$, and $P_{a}=1.2$ bars as used in the full PDE approach of Ref. [13]. Indeed, Fig. 3 exactly resembles Figs. 1 and 2 of Ref. [13]. Moreover, Table I compares the amount of vapor trapped in the bubble during collapse according to the above model to the full numerics of Ref. [13]. We find good qualitative and quantitative agreement.

Figure 4 shows the maximum temperature of an argon bubble with $R_{0}^{\mathrm{Ar}}=5 \mu \mathrm{m}, P_{a}=1.3$ bars, and $T_{0}=$ 293.15 K for different driving frequencies. The solid line gives the result according to the above model, and the dot-
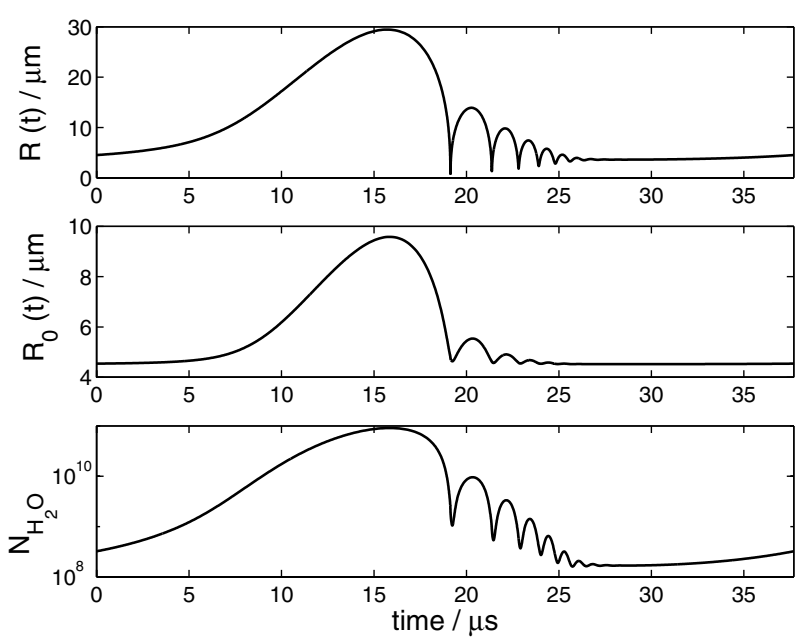

FIG. 3. $R(t), R_{0}(t)$, and $N_{\mathrm{H}_{2} \mathrm{O}}(t)$ for $f=26.5 \mathrm{kHz}, R_{0}^{\mathrm{Ar}}=$ $4.5 \mu \mathrm{m}, P_{a}=1.2$ bars, and $T_{0}=300 \mathrm{~K}$ within the presented ODE model.

ted line shows the predictions of the approach made in [5]. Rather than a monotonic increase in temperature with decreasing frequency we observe that the bubble's peak temperature goes through a maximum around $16 \mathrm{kHz}$ and $15000 \mathrm{~K}$ and then decreases again. Only at very small $f$ it increases again. This can be reasoned as follows. When the driving frequency is lowered, the bubble expands to larger maximum radius. The subsequent collapse is hence more violent, and one would observe an increase in temperature. On the other hand, the expansion to a larger maximum radius is accompanied by a larger amount of vapor being trapped during collapse, i.e., an increased heat capacity and hence a lower temperature. At low frequencies (below $16 \mathrm{kHz}$ ) the latter mechanism dominates, and thus the net effect is a decrease of the peak temperature. Note that for the parameters of Fig. 4 and a frequency $f=7.1 \mathrm{kHz}$ the mole fraction of vapor is already found to be $67.5 \%$ at collapse. The increase at very low $f$ again originates from the stronger collapse, but now the collapse of a basically pure water vapor bubble with its corresponding (temperature and time dependent) polytropic exponent. Inclusion of chemical reactions may change this very low $f$ property.

Finally, we want to calculate the model analog to our experimental results, i.e., both pulse width and light intensity. The complication is that all ions and molecules in the bubble ( $\mathrm{Ar}, \mathrm{H}_{2} \mathrm{O}$, and its eventual reaction products)

TABLE I. Comparison of the amount of vapor (mole fraction) trapped during collapse calculated from the presented model and from the full numerics of Storey and Szeri [13].

\begin{tabular}{lccccc}
\hline \hline $\begin{array}{l}R_{0}^{\mathrm{Ar}} \\
(\mu \mathrm{m})\end{array}$ & $\begin{array}{c}P_{a} \\
(\mathrm{bars})\end{array}$ & $\begin{array}{c}T_{0} \\
(\mathrm{~K})\end{array}$ & $\begin{array}{c}f \\
(\mathrm{kHz})\end{array}$ & $\begin{array}{c}\text { Szeri } \\
+ \text { Storey }(\%)\end{array}$ & $\begin{array}{c}\text { Present } \\
\text { model }(\%)\end{array}$ \\
\hline 4.5 & 1.2 & 300 & 26.5 & 14 & 14.5 \\
6 & 1.4 & 293.15 & 20.6 & 33 & 33 \\
4 & 1.32 & 293.15 & 20.6 & 27 & 23 \\
2.1 & 1.29 & 293.15 & 20.6 & 22 & 14 \\
\hline \hline
\end{tabular}




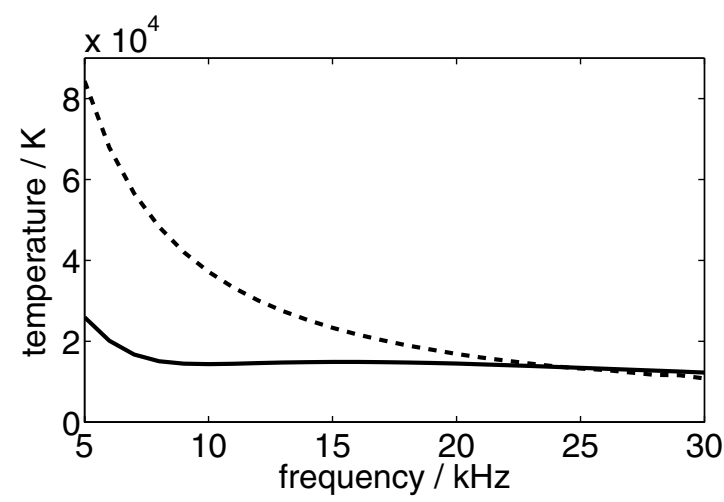

FIG. 4. Maximum temperature of an argon bubble with $R_{0}^{\mathrm{Ar}}=$ $5 \mu \mathrm{m}, P_{a}=1.3$ bars, $T_{0}=293.15 \mathrm{~K}$ (solid line: present model; dotted line: approach made in [5]).

with their different cross sections and ionization potentials contribute $[11,28]$. However, what can be said is that even at relative large frequencies oxygen and/or $\mathrm{H}_{2} \mathrm{O}$ become relevant for the light emitting process as they have the lowest ionization potential $\left(12 \mathrm{eV}\right.$ for $\mathrm{O}, 12.6 \mathrm{eV}$ for $\mathrm{H}_{2} \mathrm{O}$ in contrast to $15.8 \mathrm{eV}$ for Ar). For $f=7.1 \mathrm{kHz}$ they clearly dominate the spectrum. The preliminary calculations give pulse widths around $150 \mathrm{ps}$ and intensities of some $10^{4}$ photons per pulse, both in agreement with Fig. 1. Also the trends seen in Fig. 1 can be reproduced. This more detailed approach taking the different light emitting processes of the ions and also the chemical reactions into account is a work in progress.

In conclusion, we state that (i) at room temperatures water vapor prevents upscaling SBSL through lowering the driving frequency, (ii) ODE models of the Rayleigh-Plesset type can be extended to regard the role of water vapor $[10,12,14]$, and (iii) the competition between upscaling through lower $f$ or larger $P_{a}$ and cooling through more water vapor manifests in nonmonotonic behavior as seen in Fig. 4. At lower water temperatures around $0{ }^{\circ} \mathrm{C}$ the water vapor is only $1 / 4$ of that at room temperature and the presented model suggests that intensities $\approx 100$ times so high as here and gas temperatures in the bubble as high as $\approx 25000 \mathrm{~K}$ can be achieved. Experimental work to confirm or falsify this prediction is in progress.

It is our pleasure to acknowledge stimulating discussions with S. Hilgenfeldt. The work is part of the research program of FOM, which is financially supported by NWO.
[1] D.F. Gaitan, Ph.D. thesis, The University of Mississippi, 1990.

[2] L. A. Crum, Phys. Today 47, No. 9, 22 (1994).

[3] S. Cordry, Ph.D. thesis, The University of Mississippi, 1995.

[4] B. P. Barber et al., Phys. Rep. 281, 65 (1997).

[5] S. Hilgenfeldt and D. Lohse, Phys. Rev. Lett. 82, 1036 (1999).

[6] W. Moss, D. Clarke, and D. Young, Science 276, 1398 (1997).

[7] S. Hilgenfeldt, S. Grossmann, and D. Lohse, Nature (London) 398, 402 (1999).

[8] D. Hammer and L. Frommhold, Phys. Fluids 12, 472 (2000).

[9] K. Yasui, Phys. Rev. E 59, 1754 (1999).

[10] K. Yasui, Phys. Rev. E 56, 6750 (1997).

[11] W. C. Moss et al., Phys. Rev. E 59, 2986 (1999).

[12] A. J. Colussi and M. R. Hoffmann, J. Phys. Chem. A 103, 11336 (1999).

[13] B. D. Storey and A. J. Szeri, Proc. R. Soc. London A 456, 1685 (2000).

[14] B. D. Storey and A. J. Szeri (to be published).

[15] S.Hilgenfeldt, D. Lohse, and M. P. Brenner, Phys. Fluids 8, 2808 (1996).

[16] D. Lohse et al., Phys. Rev. Lett. 78, 1359 (1997).

[17] J. A. Ketterling and R. E. Apfel, Phys. Rev. Lett. 81, 4991 (1998).

[18] B. Gompf et al., Phys. Rev. Lett. 79, 1405 (1997).

[19] R. Pecha, B. Gompf, G. Nick, and W. Eisenmenger, Phys. Rev. Lett. 81, 717 (1998).

[20] For most features to be discussed here the latter are found to be less important as compared to the role of water vapor [13] and are therefore neglected for the time being. We also have good reason to speculate that chemical reactions may be supressed, cf. upcoming work.

[21] A. Prosperetti and A. Lezzi, J. Fluid Mech. 168, 457 (1986).

[22] C.E. Brennen, Cavitation and Bubble Dynamics (Oxford University Press, Oxford, 1995).

[23] CRC Handbook of Chemistry and Physics, edited by D. R. Lide (CRC Press, Boca Raton, 1995).

[24] V. Kamath, A. Prosperetti, and F. Egolfopoulos, J. Acoust. Soc. Am. 94, 248 (1993).

[25] J. O. Hirschfelder, C. F. Curtiss, and R. B. Bird, Molecular Theory of Gases and Liquids (Wiley, New York, 1954).

[26] G. N. Hatsopoulos and J. H. Keenan, Principles of General Thermodynamics (Wiley, New York, 1965).

[27] J. A. Fay, Molecular Thermodynamics (Addison-Wesley, Reading, MA, 1965).

[28] W. Moss and D. Young, J. Acoust. Soc. Am. 103, 3076 (1998). 